\title{
Higgs decay rate to two photons in a model with two fermiophobic-Higgs doublets
}

\author{
H. Cárdenas, ${ }^{1, *}$ A. C. B. Machado, ${ }^{2, \dagger}$ V. Pleitez, ${ }^{3, \ddagger}$ and J.-Alexis Rodriguez ${ }^{1, \S}$ \\ ${ }^{1}$ Departamento de Física, Universidad Nacional de Colombia, Bogotá, Colombia \\ ${ }^{2}$ Centro de Ciências Naturais e Humanas, Universidade Federal do ABC, Santo André, São Paulo 09210-170, Brazil \\ ${ }^{3}$ Instituto de Física Teórica-Universidade Estadual Paulista R. Dr. Bento Teobaldo Ferraz, 271, Barra Funda, São Paulo, \\ São Paulo 01140-070, Brazil
}

(Received 12 December 2012; published 22 February 2013)

\begin{abstract}
We consider a three Higgs doublet model with an $S_{3}$ symmetry in which beside the standard model-like doublet, there are two fermiophobic doublets. Due to the new charged scalars, there is an enhancement in the two-photon decay, while the other channels have the same decay widths as the standard model neutral Higgs. The fermiophobic scalars are mass degenerated unless soft terms breaking the $S_{3}$ symmetry are added.
\end{abstract}

DOI: 10.1103/PhysRevD.87.035028

PACS numbers: 12.60.Fr, 12.15.-y

\section{INTRODUCTION}

Recently, a new resonance which is compatible with the Higgs boson of the standard model (SM) with a mass of $125 \mathrm{GeV}$ has been discovery at the LHC [1]. As it is well known in the context of that model, nothing constraints the number of fermion generations; although, since the LEP data, we know that there exist only three sequential generations of quarks and leptons. This triplication may also exist in the scalar sector since here again, nothing constraints the number of Higgs scalar multiplets and, in particular, the number of Higgs doublets is a free parameter in the model, although one of them is enough to accommodate vector and fermion masses and their mixing. In this vein the multi-Higgs extensions of the standard model are among the most motivated new physics scenarios. Generally, these models have scalar mediated flavor changing neutral currents. Even in the simplest case, the twoHiggs doublet models have several possibilities to control those effects [2]. Three-Higgs doublet models [3] have not been considered with the same details as those of the twoHiggs doublet case. This is not a surprise since in this case the analysis of the scalar potential is much more complicated. However, discrete symmetries may simplify the scalar potential; for example, the $A_{4}$ symmetry has been considered in Ref. [4]. Recently, it was shown that the $S_{3}$ symmetry is very efficient to constraint the scalar potential allowing us to obtain the mass spectra and the matrix, which diagonalize the mass square matrices [5]. The symmetries for the two-Higgs doublet model was obtained in Ref. [6] and in the case of three-Higgs doublet models in Ref. [7].

If one or more extra Higgs doublets do exist in nature, it seems that their existence is due to some reason that allows us to explain something else that could not be explained by

\footnotetext{
*hjcardenasr@unal.edu.co

†ana@ift.unesp.br

*vicente@ift.unesp.br

§arodriguezl@unal.edu.co
}

the minimal model, i.e., with only one Higgs doublet. On one hand, it is possible that extra scalars may explain the mass spectra and mixing in the fermion sectors [8], or on the other hand, it is possible that the extra Higgs doublets may help to understand the observed dark matter. In the latter case the extra Higgs bosons have to be of the fermiophobic type [9]. The fermiophobic Higgs boson is defined as the following: all the fermion couplings to the Higgs boson are set to zero and the bosonic couplings are the same as in the standard model. This, in fact, has been already considered in the case of one fermiophobic doublet-Higgs model [10]. Here we will consider the LHC phenomenology of the three doublet model with $S_{3}$ symmetry [11] which was put forward in Ref. [5] and which has two fermiophobic doublets.

The outline of this paper is as follows: In the next section we review the main feature of the three scalars model. In Sec. III we give the interactions of the model. In Sec. III A we give the Yukawa interactions, in Sec. III B the gauge interactions, while in Secs. III C and III D we write down explicitly the trilinear interactions. In Sec. IV we show the decay rate into two photon of the SM-like neutral scalar. We devote Sec. V for our conclusions. In the Appendix we show how the mass spectra are modified if we add soft terms to the scalar potential.

\section{THE SCALAR SECTOR}

Let us consider an extension of the SM electroweak theory which consists in adding two extra scalars, $S U(2)_{L}$ doublets, with $Y=+1$. The three scalar doublets are in a singlet $S$ and a doublet $D$ of $S_{3}$. The $S U(2)_{L} \otimes$ $U(1)_{Y} \otimes S_{3}$ invariant scalar potential is given by

$$
V=V(D, S)+V(D, S)_{\mathrm{soft}},
$$




$$
\begin{aligned}
V(D, S)= & \mu_{s}^{2} S^{\dagger} S+\mu_{d}^{2}\left[D^{\dagger} \otimes D\right]_{1}+\lambda_{1}\left(\left[D^{\dagger} \otimes D\right]_{1}\right)^{2}+\lambda_{2}\left[\left(D^{\dagger} \otimes D\right)_{1^{\prime}}\left(D^{\dagger} \otimes D\right)_{1^{\prime}}\right]_{1} \\
& +\lambda_{3}\left[\left(D^{\dagger} \otimes D\right)_{2}\left(D^{\dagger} \otimes D\right)_{2}\right]+\lambda_{4}\left(S^{\dagger} S\right)^{2}+\lambda_{5}\left[D^{\dagger} \otimes D\right]_{1} S^{\dagger} S+\lambda_{6} S^{\dagger}\left[D^{\dagger} \otimes D\right]_{1} S \\
& +\left\{\lambda_{7}\left[\left(S^{\dagger} \otimes D\right)_{2}\left(D^{\dagger} \otimes S\right)_{2}\right]_{1}+\lambda_{8}\left[\left(S^{\dagger} \otimes D\right)_{2}\left(D^{\dagger} \otimes D\right)_{2}\right]_{1}+\text { H.c. }\right\},
\end{aligned}
$$

and $V_{\text {soft }}$ denote soft terms breaking $S_{3}$ symmetry explicitly; see Ref. [5]. The effects of the soft terms on the scalar masses are considered in the Appendix.

There are two ways to build the singlet $S$ and the doublet $D$ which are not equivalent. In the first one, we call model A, the reducible triplet representation of the discrete symmetry $S_{3}: \mathbf{3}=\left(H_{1}, H_{2}, H_{3}\right)$ with the usual notation $H_{i}=$ $\left(H^{+} H_{i}^{0}\right)^{T}$ in which $H_{i}^{0}=(1 / \sqrt{2})\left(v+\eta^{0}+i A^{0}\right)$. This reducible representation is the direct sum of one singlet and one doublet $S_{3}=\mathbf{2}+\mathbf{1} \equiv D+S$, where $S$ and $D$ are give by

$$
\begin{aligned}
S & =\frac{1}{\sqrt{3}}\left(H_{1}+H_{2}+H_{3}\right) \sim \mathbf{1}, \\
D & \equiv\left(D_{1}, D_{2}\right)=\left[\frac{1}{\sqrt{6}}\left(2 H_{1}-H_{2}-H_{3}\right), \frac{1}{\sqrt{2}}\left(H_{2}-H_{3}\right)\right] \sim \mathbf{2},
\end{aligned}
$$

and the other way, we denote model $\mathrm{B}$, is such that

$$
S=H_{1} \sim \mathbf{1}, \quad D=\left(H_{2}, H_{3}\right) \sim \mathbf{2} .
$$

We also impose a vacuum alignment in each case: $(v, v, v)$ and $\left(v_{\mathrm{SM}}, 0,0\right)$ in model $\mathrm{A}$ and $\mathrm{B}$, respectively. This vacuum alignment gives a global and stable minimum of the scalar potential if other conditions are satisfied, too [5]. In both cases the constraint equations reduce to $\mu_{s}^{2}=$ $-\lambda_{4} v_{\mathrm{SM}}^{2}$ which implies that $\lambda_{4}>0$. The difference is that $3 v^{2}=v_{\mathrm{SM}}^{2}$ in the model $\mathrm{A}$, and $v_{1}^{2}=v_{\mathrm{SM}}^{2}$, in model B; see Ref. [5] for details.

With the scalar potential in Eq. (2) in model A the mixing matrix in all the scalar, pseudoscalar, and charged scalar sectors is given by the tribimaximal matrix

$$
U_{\mathrm{TBM}}=\left(\begin{array}{ccc}
\frac{1}{\sqrt{3}} & -\sqrt{\frac{2}{3}} & 0 \\
\frac{1}{\sqrt{3}} & \frac{1}{\sqrt{6}} & -\frac{1}{\sqrt{2}} \\
\frac{1}{\sqrt{3}} & \frac{1}{\sqrt{6}} & \frac{1}{\sqrt{2}}
\end{array}\right),
$$

and the masses are the following: in the $C P$ even sector

$$
m_{h_{1}}^{2}=\lambda_{4} v_{\mathrm{SM}}^{2}, \quad m_{h_{2}}^{2}=m_{h_{3}}^{2}=\mu_{d}^{2}+\frac{1}{2} \bar{\lambda}^{\prime} v_{\mathrm{SM}}^{2}
$$

where $\bar{\lambda}^{\prime}=\lambda_{5}+\lambda_{6}+2 \lambda_{7}$, and denoting as $h_{i}^{0}$ the mass eigenstates, we have $\eta_{i}^{0}=\left(U_{\mathrm{TBM}}\right)_{i j} h_{j}^{0}$, where $U_{\mathrm{TBM}}$ is given in (5). The scalar $h_{1}^{0}$ can be identified with the standard model Higgs scalar.

In the $C P$-odd neutral scalars sector, we obtain the following masses:

$$
m_{a_{1}}^{2}=0, \quad m_{a_{2}}^{2}=m_{a_{3}}^{2}=\mu_{d}^{2}+\frac{1}{2} \bar{\lambda}^{\prime} v_{\mathrm{SM}}^{2} .
$$

Denoting $a_{i}^{0}$ the pseudoscalar mass eigenstates, we have $A_{i}^{0}=\left(U_{\mathrm{TBM}}\right)_{i j} a_{j}^{0}$.

Similarly in the charged scalars sector we obtain the following masses:

$$
m_{c_{1}}^{2}=0, \quad m_{c_{2}}^{2}=m_{c_{3}}^{2}=\frac{1}{4}\left(2 \mu_{d}^{2}+\lambda_{5} v_{\mathrm{SM}}^{2}\right),
$$

and denoting $\mathrm{H}_{i}^{+}$denote the charged scalar symmetry eigenstates and $h_{i}^{+}$the respective mass eigenstates, we have $H_{i}^{+}=\left(U_{\mathrm{TBM}}\right)_{i j} h_{j}^{+}$.

In model A the $S U(2)$ doublets can be written in terms of the mass eigenstates using the mixing matrix em Eq. (5), resulting in

$$
\begin{gathered}
S=\left(\begin{array}{c}
h_{1}^{+} \\
\frac{1}{\sqrt{2}}\left(3 v+h_{1}^{0}+i a_{1}^{0}\right)
\end{array}\right), \quad D_{1}=-\left(\begin{array}{c}
h_{2}^{+} \\
\frac{1}{\sqrt{2}}\left(h_{2}^{0}+i a_{2}^{0}\right)
\end{array}\right), \\
D_{2}=-\left(\begin{array}{c}
h_{3}^{+} \\
\frac{1}{\sqrt{2}}\left(h_{3}^{0}+i a_{3}^{0}\right)
\end{array}\right) .
\end{gathered}
$$

However, in model B the mass matrices are diagonal, i.e., there is no mixing in each charge sector. In general, the eigenvalues are equal to those in Eq. (6) for $C P$ even sector, Eq. (7) for $C P$ odd sector, and Eq. (8) for the charged scalar sector, respectively.

Notice that the mass degeneracy in the fermiophobic sector is a prediction of the $S_{3}$ symmetry but there may be accidental mass degeneracy with the SM-like Higgs boson, too.

The possibility that two mass degenerated Higgs bosons with mass near the $125 \mathrm{GeV}$ has been discussed in literature [12-16]. The main difference with the present model is that two of the Higgs doublets are fermiophobic; they do not interact with quarks or leptons at tree level. On the other hand, they can be produced in accelerators like the LEP by the Higgstrahlung mechanism $e^{+} e^{-} \rightarrow Z^{*} \rightarrow Z X$ or in hadronic colliders $q q^{\prime} \rightarrow V V \rightarrow X$ where $X$ denotes any neutral scalar. Moreover, since they are fermiophobic scalars they do not decay into fermions and they behave as invisible Higgses. Bounds on the masses of femiophobic Higgs boson in the diphoton decay channel exclude this sort of scalars in the ranges $110-118 \mathrm{GeV}$ and 119.5 and $121 \mathrm{GeV}$ [17]. This is the case of the fermiophobic Higgs in the present model. Moreover, the decay $Z Z \rightarrow 4 l$ is exactly the same as in the SM since only one of the neutral scalar (the one which is not fermiophobic, $h_{1}$ ) contributes to these decays. 


\section{INTERACTIONS}

\section{A. The Yukawa sector}

The Yukawa interactions are equal in both models when vacua are aligned as before. Only one of the doublets interacts with quarks and leptons and the other two are fermiophobic doublets. In the lepton sector all lepton fields transform as singlet under $S_{3}$ and for this reason they only interact with the singlet $S$ :

$$
-\mathcal{L}_{l}=\bar{L}_{i L}^{\prime} G_{i j}^{l} S l_{j R}^{\prime}+\bar{L}_{i L}^{\prime} G_{i j}^{\nu} \tilde{S} \nu_{j R}^{\prime}+\text { H.c., }
$$

where the prime fields denote symmetry eigenstates which are written in terms of the mass (unprimed) fields by using unitary matrices:

$$
\begin{aligned}
l_{i L}^{\prime}=\left(U_{L}^{l}\right)_{i j} l_{j L}, & l_{i R}^{\prime}=\left(U_{R}^{l}\right)_{i j} l_{j R}, \\
\nu_{i L}^{\prime}=\left(U_{L}^{\nu}\right)_{i j} \nu_{j L}, & l_{i R}^{\prime}=\left(U_{R}^{l}\right)_{i j} l_{j R} .
\end{aligned}
$$

The Yukawa interactions written in terms of the mass eigenstates are

$$
\begin{aligned}
-\mathcal{L}_{l}= & \bar{\nu}_{i L} \frac{\hat{M}_{i}^{l}}{v_{\mathrm{SM}}}\left(V_{\mathrm{PMNS}}\right)_{i j} l_{j R} h_{1}^{+}+\bar{l}_{i L} \frac{\hat{M}_{i}^{l}}{v_{\mathrm{SM}}} l_{j R}\left[1+\frac{h_{1}^{0}+i a_{1}^{0}}{\sqrt{2}}\right] \\
& +\bar{l}_{i L} \frac{\hat{M}_{i}^{\nu}}{v_{\mathrm{SM}}}\left(V_{\mathrm{PMNS}}\right)_{i j} \nu_{j R} h_{1}^{-} \\
& +\bar{\nu}_{i L} \frac{\hat{M}_{i}^{v}}{v_{S M}} \nu_{i R}\left[1+\frac{h_{1}^{0}+i a_{1}^{0}+}{\sqrt{2}}\right]+\text { H.c., }
\end{aligned}
$$

where we have defined $V_{\text {PMNS }}=U_{L}^{l \dagger} U_{L}^{\nu}$.

Similarly, all quarks fields are singlet under $S_{3}$, hence as in the lepton case, they only interact with the singlet $S$ :

$$
-\mathcal{L}_{q}=\bar{Q}_{i L}^{\prime} G_{i j}^{u} \tilde{S} u_{j R}^{\prime}+\bar{Q}_{i L}^{\prime} G_{i j}^{d} S d_{j R}^{\prime}+\text { H.c., }
$$

and using

$$
\begin{array}{ll}
u_{i L}^{\prime}=\left(U_{L}^{u}\right)_{i j} u_{j L}, & u_{i R}^{\prime}=\left(U_{R}^{u}\right)_{i j} l_{j R}, \\
d_{i L}^{\prime}=\left(U_{L}^{d}\right)_{i j} d_{j L}, & d_{i R}^{\prime}=\left(U_{R}^{d}\right)_{i j} d_{j R},
\end{array}
$$

we write the Yukawa interactions in terms of the quark mass eigenstates

$$
\begin{aligned}
-\mathcal{L}_{q}= & \bar{u}_{i L} \frac{\hat{M}_{i}^{d}}{v_{\mathrm{SM}}}\left(V_{\mathrm{CKM}}\right)_{i j} d_{j R} h_{1}^{+}+\bar{d}_{i L} \frac{\hat{M}_{i}^{d}}{v_{\mathrm{SM}}}\left[1+\frac{h_{1}^{0}}{\sqrt{2}}\right] d_{i R} \\
& +\bar{d}_{i L} \frac{\hat{M}_{i}^{u}}{v_{S M}}\left(V_{\mathrm{CKM}}\right)_{i j} u_{j R} h_{1}^{-} \\
& +\bar{u}_{i L} \frac{\hat{M}_{i}^{u}}{v_{\mathrm{SM}}} u_{i R}\left[1+\frac{h_{1}^{0}}{\sqrt{2}}\right]+\text { H.c., }
\end{aligned}
$$

where we have defined $V_{\mathrm{CKM}}=U_{L}^{u \dagger} U_{L}^{d}$. Above $\hat{M}$ denotes diagonal mass matrices in the respective charge sector.

As in the standard model the masses and the $V_{\mathrm{CKM}}$ and $V_{\text {PMNS }}$ mixing matrices can be accommodated but their values are not explained.

\section{B. Gauge-scalar interactions}

In this sector, when the scalar doublets are written in terms of the mass eigenstates, only one of the scalar doublets contribute to the vector boson masses as in the SM. The $S U(2)_{L} \otimes U(1)_{Y} \otimes S_{3}$ invariant gauge interactions are

$$
\begin{aligned}
\mathcal{L}_{\text {gauge }}= & \left(\mathcal{D}_{\mu} S\right)^{\dagger}\left(\mathcal{D}^{\mu} S\right)+\left(\mathcal{D}_{\mu} D\right)^{\dagger}\left(\mathcal{D}^{\mu} D\right) \\
= & \left(\mathcal{D}_{\mu} H_{1}\right)^{\dagger}\left(\mathcal{D}^{\mu} H_{1}\right)+\left(\mathcal{D}_{\mu} H_{2}\right)^{\dagger}\left(\mathcal{D}^{\mu} H_{2}\right) \\
& +\left(\mathcal{D}_{\mu} H_{3}\right)^{\dagger}\left(\mathcal{D}^{\mu} H_{3}\right),
\end{aligned}
$$

where $S, D$, or $H_{i}$ are symmetry eigenstates. Using the first line and the fields in Eqs. (9) and (A5), we can write the Higgs scalar gauge interactions in terms of the mass eigenstates:

$$
\begin{aligned}
\mathcal{L}_{\text {gauge }}= & \left(\mathcal{D}_{\mu} h_{1}\right)^{\dagger}\left(\mathcal{D}^{\mu} h_{1}\right)+\left(\mathcal{D}_{\mu} h_{2}\right)^{\dagger}\left(\mathcal{D}^{\mu} h_{2}\right) \\
& +\left(\mathcal{D}_{\mu} h_{3}\right)^{\dagger}\left(\mathcal{D}^{\mu} h_{3}\right),
\end{aligned}
$$

where $h_{i}=\left[h_{i}^{+},\left(h_{i}^{0}+i a_{i}^{0}\right) / \sqrt{2}\right]^{T}, i=1,2,3$ are the $S U(2)$ doublets written in terms of the mass eigenstates. We have omitted the mass term, i.e., the vacuum expectation value in $h_{1}$. The covariant derivative $\mathcal{D}_{\mu}$ is the same of the standard model.

\section{Trilinear interactions in model A}

The trilinear interactions in model A with or without the soft terms (see the Appendix) are as follows:

$$
\frac{v_{\mathrm{SM}}}{2 \sqrt{3}}\left[\lambda_{4} h_{1}^{-} h_{1}^{+}+\lambda_{5}\left(h_{2}^{-} h_{2}^{+}+h_{3}^{-} h_{3}^{+}\right)\right]\left(h_{1}^{0}-i a_{1}^{0}\right) .
$$

In the same way for the second scalar, we have

$$
\frac{v_{\mathrm{SM}}}{2 \sqrt{3}}\left[\left(\lambda_{6}+\lambda_{7}\right) h_{1}^{-} h_{2}^{+}-\lambda_{8}\left(h_{2}^{-} h_{2}^{+}+h_{3}^{-} h_{3}^{+}\right)\right]\left(-h_{2}^{0}+i a_{2}^{0}\right) .
$$

Note that the vertex with $h_{1}^{-} h_{3}^{+}$does not exist. Finally, for the third scalar

$$
\frac{v_{\mathrm{SM}}}{2 \sqrt{3}}\left[-\left(\lambda_{6}+\lambda_{7}\right) h_{1}^{-} h_{3}^{+}+\lambda_{8} h_{2}^{-} h_{3}^{+}\right]\left(-h_{3}^{0}+i a_{3}^{0}\right),
$$

and in this case, the vertex with $h_{1}^{-} h_{2}^{+}$that does not exist.

In the neutral scalar and pseudoscalar sector we have (up to a factor $v_{\mathrm{SM}} / 2 \sqrt{3}$ )

$$
\begin{aligned}
& {\left[\lambda_{4}\left(h_{1}^{0} h_{1}^{0}+a_{1}^{0} a_{1}^{0}\right)+\lambda_{5}\left(h_{2}^{0} h_{2}^{0}+h_{3}^{0} h_{3}^{0}+a_{2}^{0} a_{2}^{0}+a_{3}^{0} a_{3}^{0}\right)\right]} \\
& \quad \times\left(h_{1}^{0}-i a_{1}^{0}\right)-\left[\left(\lambda_{6}+\lambda_{7}\right)\left(h_{1}^{0}+i a_{1}^{0}\right)\left(h_{2}^{0}+i a_{2}^{0}\right)\right. \\
& \left.\quad+\lambda_{8}\left(h_{2}^{0} h_{2}^{0}+a_{2}^{0} a_{2}^{0}-h_{3}^{0} h_{3}^{0}-a_{3}^{0} a_{3}^{0}\right)\right]\left(-h_{2}^{0}+i a_{2}^{0}\right) \\
& \quad-\left[\left(\lambda_{6}+\lambda_{7}\right)\left(-h_{1}^{0} h_{3}^{0}+a_{1}^{0} a_{3}^{0}+i\left(a_{1}^{0} h_{3}^{0}-a_{3}^{0} h_{1}^{0}\right)\right)\right. \\
& \left.\quad-2 \lambda_{8}\left(a_{2}^{0} a_{3}^{0}+h_{2}^{0} h_{3}^{0}\right)\right]\left(-h_{3}^{0}+i a_{3}^{0}\right) .
\end{aligned}
$$




\section{Trilinear interactions in model B}

\section{Without the soft terms}

In model B without the soft terms we have the following trilinear interactions:

$$
\begin{gathered}
\frac{v_{\mathrm{SM}}}{2}\left[\lambda_{4} h_{1}^{-} h_{1}^{+}+\lambda_{5}\left(h_{2}^{+} h_{2}^{-}+h_{3}^{+} h_{3}^{-}\right)\right]\left(h_{1}^{0}-i a_{1}^{0}\right), \\
\frac{v_{\mathrm{SM}}}{2 \sqrt{2}}\left(\lambda_{6}+\lambda_{7}\right) h_{1}^{-} h_{2}^{+}\left(-h_{2}^{0}+i a_{2}^{0}\right),
\end{gathered}
$$

and

$$
\frac{v_{\mathrm{SM}}}{2 \sqrt{2}}\left(\lambda_{6}+\lambda_{7}\right) h_{1}^{-} h_{3}^{+}\left(h_{3}^{0}+i a_{3}^{0}\right) .
$$

In the neutral sector (up to a factor $v_{\mathrm{SM}} / 2$ )

$$
\begin{aligned}
& {\left[\frac{\lambda_{4}}{2}\left(h_{1}^{0} h_{1}^{0}+a_{1}^{0} a_{1}^{0}\right)+\left(\lambda_{5}+\lambda_{6}+\lambda_{7}\right)\right.} \\
& \left.\quad \times\left(h_{2}^{0} h_{2}^{0}+h_{3}^{0} h_{3}^{0}+a_{2}^{0} a_{2}^{0}+a_{3}^{0} a_{3}^{0}\right)\right]\left(h_{1}^{0}-a_{1}^{0}\right) \\
& \quad+\frac{1}{4}\left(\lambda_{6}+\lambda_{7}\right)\left(h_{1}^{0}+i a_{1}^{0}\right)\left(h_{2}^{0}-i a_{2}^{0}\right)\left(h_{2}^{0}+i a_{2}^{0}\right) \\
& \quad+\frac{1}{4}\left(\lambda_{6}+\lambda_{7}\right)\left(h_{1}^{0}+i a_{1}^{0}\right)\left(h_{3}^{0}-i a_{3}^{0}\right)\left(h_{3}^{0}+i a_{3}^{0}\right) .
\end{aligned}
$$

\section{With soft terms}

In model B (see the Appendix) when the soft terms are included we have the following trilinear interactions:

$$
\begin{aligned}
\frac{v_{\mathrm{SM}}}{2}[ & \left.\lambda_{4} h_{1}^{-} h_{1}^{+}+\lambda_{5}\left(h_{2}^{+} h_{2}^{-}+h_{3}^{+} h_{3}^{-}\right)\right]\left(h_{1}^{0}+i a_{1}^{0}\right), \\
& \frac{v_{\mathrm{SM}}}{2 \sqrt{2}}\left(\lambda_{6}+\lambda_{7}\right) h_{1}^{-}\left(h_{3}^{+}-h_{2}^{+}\right)\left(-h_{2}^{0}+i a_{2}^{0}\right), \\
& \frac{v_{\mathrm{SM}}}{2 \sqrt{2}}\left(\lambda_{6}+\lambda_{7}\right) h_{1}^{-}\left(h_{3}^{+}+h_{2}^{+}\right)\left(-h_{3}^{0}-i a_{3}^{0}\right),
\end{aligned}
$$

and, up to a factor $v_{\mathrm{SM}} / \sqrt{2}$

$$
\begin{aligned}
& \frac{v_{\mathrm{SM}}}{2 \sqrt{2}}\left[\lambda_{4}\left(h_{1}^{0} h_{1}^{0}+a_{1}^{0} a_{1}^{0}\right)+2\left(\lambda_{5}+\lambda_{6}+\lambda_{7}\right)\right. \\
& \left.\quad \times\left(h_{2}^{0} h_{2}^{0}+h_{3}^{0} h_{3}^{0}+a_{2}^{0} a_{2}^{0}+a_{3}^{0} a_{3}^{0}\right)\right]\left(h_{1}^{0}-a_{1}^{0}\right) .
\end{aligned}
$$

We see that model A differs from the model B only in the trilinear (and quartic but we have not shown they here) interactions. Model B also has different scalar-scalar interactions depending if we add or not the soft term to the scalar potential. Thus, those possibilities may be distinguished when Higgs self-couplings are measured at the LHC [18].

\section{RESULTS AND DISCUSSIONS}

We can explore the phenomenology associated to the Higgs sector of this model under some basic assumptions.
We are going to consider only the model A in this section without soft terms added although they do not modify our results. If $h_{1}^{0}$ is the SM-like Higgs boson, its mass has to be near $125 \mathrm{GeV}$; it implies $\lambda_{4}=0.26$. From Eqs. (6)-(8), we obtain typical values of the scalar bosons masses in both models, respectively. We can evaluate the decay channels of the neutral $C P$-even Higgs $h_{1}^{0}$ in a mass range around $125 \mathrm{GeV}$, and we can compare the branching fractions with the SM results. The Higgs sector depends on the Higgs mass spectrum which is parametrized in terms of $\lambda_{5}, \lambda_{6}$, $\lambda_{7}, \mu_{d}^{2}$ in the model A. On the other hand, the fermiophobic Higgs fields $h_{2,3}$ only interact through the trilinear terms already mentioned, and if we assume these Higgs bosons with a mass bigger than $125 \mathrm{GeV}$, then they only are going to contribute to the $h \rightarrow \gamma \gamma$ and $h \rightarrow \gamma Z$. It is interesting that the $h \rightarrow \gamma \gamma$ decay because there is an excess of events above the SM predictions. We are going to focus on this decay mode because in the other decay channels there are not significant contributions respect to the SM expectations. It is useful to define a reduced signal rate $R$ relative to the expected signal of the SM Higgs boson [19]

$$
R_{\gamma \gamma}=\frac{\sigma\left(p p \rightarrow h_{1}^{0}\right)}{\sigma\left(p p \rightarrow h_{\mathrm{SM}}\right)} \frac{\mathrm{BR}\left(h_{1}^{0} \rightarrow \gamma \gamma\right)}{\mathrm{BR}\left(h_{\mathrm{SM}} \rightarrow \gamma \gamma\right)},
$$

where the first factor is associated with the production mechanism which in our case is mainly through the gluon-gluon fusion, and the second factor is the reduced branching fraction for the channel under consideration. In model A, the first factor will be one because there are not any new contributions from the fermiophobic Higgs bosons interactions to the Higgs production; the new Higgs bosons do not couple to the quarks. Therefore, $R_{\gamma \gamma}$ is the reduced branching fraction. In the $h_{1}^{0} \rightarrow \gamma \gamma$ decay channel there are contributions in the loop from the couplings $h_{1}^{0} h_{2,3}^{+} h_{2,3}^{-}$which are proportional to $\lambda_{5}$. There are experimental reports from CMS and ATLAS collaborations to the $R_{\gamma \gamma}$ fraction in the $\gamma \gamma$ mode and the combined results imply in $R_{\gamma \gamma}=1.66 \pm 0.36$ [19], which we are going to use to constraint the model A parameters. In Fig. 1, we have plotted the $R_{\gamma \gamma}$ fraction vs the parameter $\mu_{d}$ using $\lambda_{5}=1$ and $\lambda_{6}=\lambda_{7}=0$. The parameters $\lambda_{6,7}$ are involved in the Higgs boson masses while $\lambda_{5}$ is also appearing in the trilinear couplings. From Fig. 1, there is an allowed region for $\mu_{d}$ between $65-260 \mathrm{GeV}$ and $355-435 \mathrm{GeV}$ and excluded $260-355 \mathrm{GeV}$; these intervals correspond to fermiophobic Higgs boson masses of $m_{h_{2}}=m_{a_{2}}=$ $185-312 \mathrm{GeV}$ and $m_{c_{2}}=130-221 \mathrm{GeV}$ in the first allowed interval and $m_{h_{2}}=m_{a_{2}}=279-395 \mathrm{GeV}$ and $m_{c_{2}}=279-331 \mathrm{GeV}$ in the second one. Here we should emphasize that in the model A the fermiophobic Higgs fields are mass degenerate. In Fig. 2, we have to make a contour plot in the plane $\lambda_{5}-\mu_{d}$ using the experimental value of the reduced branching fraction $R_{\gamma \gamma}$ in order to explore the space parameter of $\lambda_{5}$. The allowed region is 


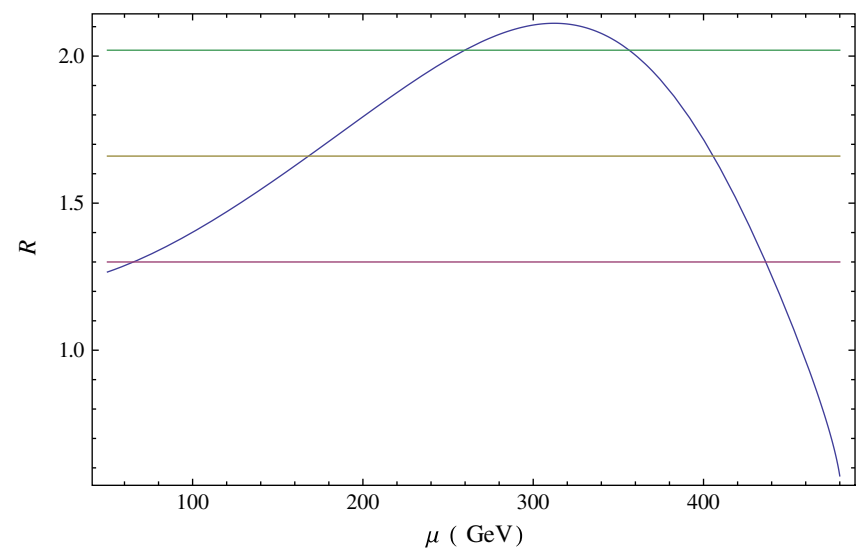

FIG. 1 (color online). The reduced branching fraction $R_{\gamma \gamma}$ vs $\mu_{d}$ using $\lambda_{5}=1$ and $\lambda_{6}=\lambda_{7}=0$ in the model A. The solid lines correspond to the combined experimental value $R_{\gamma \gamma}=$ $1.66 \pm 0.36$ [19]. An excluded area is found around 260$355 \mathrm{GeV}$.

the light colored region and there are excluded areas around and in the middle of the contour which is the white area. Some Higgs boson masses acquired from Fig. 2 are in Table I. A brief comment about the parameters $\lambda_{6,7}$ is that their values are not affecting the regions obtained because they only appear in the expression of the fermiophobic Higgs boson masses in the loop.

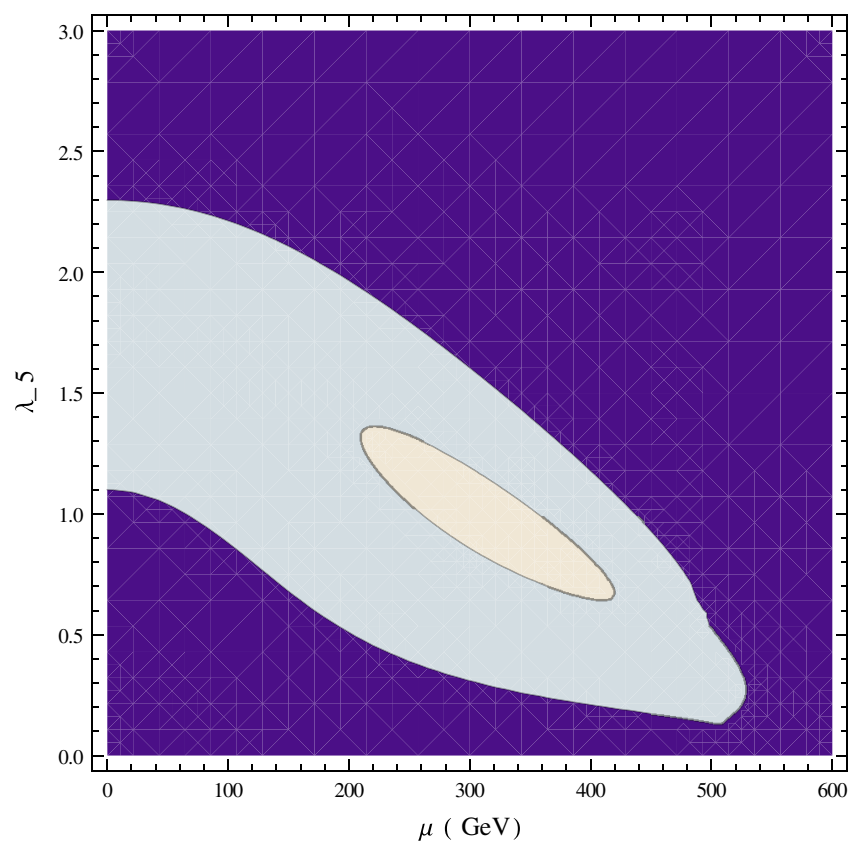

FIG. 2 (color online). Contour plot $\lambda_{5}-\mu_{d}$ with $\lambda_{6}=\lambda_{7}=0$ constrained by $R_{\gamma \gamma}=1.66 \pm 0.36$. The allowed region is the light region with a white excluded area inside. Some points of this plot are shown explicitly in Table I with their associated Higgs boson masses.
TABLE I. Some points from Fig. 2 and the associated Higgs boson masses. As we can see from Eqs. (6) and (7), we have $m_{h_{2}}=m_{a_{2}}$.

\begin{tabular}{lccc}
\hline \hline$\lambda_{5}$ & $\mu_{d}(\mathrm{GeV})$ & $m_{h_{2}}(\mathrm{GeV})$ & $m_{c_{2}}(\mathrm{GeV})$ \\
\hline 1 & 0 & 175 & 123 \\
2.3 & 0 & 264 & 186 \\
1.3 & 203 & 283 & 200 \\
0.6 & 410 & 433 & 306 \\
0.11 & 511 & 514 & 363 \\
\hline \hline
\end{tabular}

A brief comment about the production of the fermiophobic Higgs bosons should be addressed. The recent discovery of a Higgs-like boson at the LHC does not rule out the possibility of a Higgs boson decaying into a channel with invisible decay products as in our case the $h_{1}^{0}$ into $h_{2,3}^{0} h_{2,3}^{0}$ or $h_{2,3}^{ \pm} h_{2,3}^{ \pm}$[20]. The most important channel for the detection of this invisible mode is vector boson fusion since it has a large cross section, but it also has large systematic uncertainties and it is difficult to estimate the QCD background. Another option is the associated production channel $W h^{0}$ or $Z h^{0}$, however, the $W h^{0}$ channel is diluted by the inclusive $W$ background, which makes it difficult to analyze; instead, the $Z h$ channel is more promising [20]. On the other hand, these fermiophobic Higgs bosons are already candidates to dark matter and constraints from their production through channels like $h_{2,3}^{0} h_{2,3}^{0} \rightarrow h_{1}^{0} \rightarrow \gamma \gamma$ will be expected.

\section{CONCLUSIONS}

Fermiophobic Higgs fields only interact with themselves and to other scalar and vector bosons or active multiplets; they have been called inert [21] or dark [22] Higgses and they may transform under the gauge symmetries of the SM in nontrivial way as doublets $[9,21]$, or in a trivial way, i.e., singlets [23]. They have been considered as solutions to the hierarchy problem or/and as a good cold dark matter candidates [21,23]. Here, we have built up an extension of the SM adding two extra doublet scalars and using a $S_{3}$ symmetry. There are two ways to build the singlet and the doublet of $S_{3}$; we called them model A and B (see the Appendix). The models are different in their trilinear and cuartic interactions but they have a dark degenerate scalar spectrum. The interactions of the SM-like Higgs boson are identical to the SM. Therefore, the only effect from the dark scalars is in the one loop processes like the Higgs boson into two photon decay. We have evaluated the reduced fraction $R_{\gamma \gamma}$ in order to get constraints for the parameters $\lambda_{5}$ and $\mu_{d}$ of the model A. On the other hand, models A and B are predicting the same $h_{1} \rightarrow \gamma \gamma$ width decay but they are not equivalent in the invisible modes. In general a fermiophobic neutral scalar decays to $W$ and $Z$ bosons proceeds as in the SM, while the decay to photons proceed via $W, h^{ \pm}$loops, since its decays to 
photons via fermion loops are excluded. If this were the case for the doublet of the standard model, i.e., if the fermion masses have a different origin from that of the gauge boson, it is excluded at $95 \%$ confidence level in the mass range 110-194 GeV, and at 99\% confidence level in the mass ranges 110-124.5, 127-147.5, and $155-180 \mathrm{GeV}$ [24]. Notice that in this case there is a small window around 124.5-127 GeV. Hence, the constraints above are not directly applicable to the model considered in this work that has the usual Higgs doublet plus two dark doublets. However, in the present model, fermions masses arise from the Higgs doublet, which also contributes to the gauge boson masses but the latter particles also have contributions from the dark doublets.

Note that for the calculations performed in this work we consider that $\lambda_{6}=\lambda_{7}=0$, since they do not contribute in the photon-photon loop; however, as a result of this assumption, the charged boson is lighter than the neutral boson; some values are shown in Table I. As a consequence the neutral scalar is not a good candidate for dark matter. However, if we consider that good candidates for dark matter must satisfy the relation $m_{c}^{2}-m_{h}^{2} \geq 0$ implying $-\frac{\mu^{2}}{v_{\mathrm{SM}}^{2}}-\frac{1}{2} \lambda_{5}-\left(\lambda_{6}+2 \lambda_{7}\right) \geq 0$, it is easy to see that, for example, with $\mu_{d}=82 \mathrm{GeV}, \lambda_{5}=1, \lambda_{6}=-0.82$, and $\lambda_{7}=0$ we obtained $m_{h}=110 \mathrm{GeV}$ and $m_{c}=136$; thus, we can have a dark matter scenario in these models by imposing that the inequality must be satisfied.

\section{ACKNOWLEDGMENTS}

H.C. and J.A.R. acknowledge the hospitality of IFT, Sao Paulo were this work was finished. One of the authors (A. C. B. M.) was supported by CAPES and (V.P.) was partially supported by $\mathrm{CNPq}$ and FAPESP. H.C. and J.A.R. were partially supported by Grant No. 14844 DIB-UNAL.

\section{APPENDIX: SCALAR MASSES WITH SOFT TERMS IN THE SCALAR POTENTIAL}

The mass degeneracy above is due to a residual symmetry that can be broken, if necessary, by including the soft terms:

$V(D, S)_{\text {soft }}=\mu_{22}^{2} H_{2}^{\dagger} H_{2}+\mu_{33}^{2} H_{3}^{\dagger} H_{3}+\left(\mu_{23}^{2} H_{2}^{\dagger} H_{3}+\right.$ H.c. $)$.

In model $\mathrm{A}$, taken into account the soft terms with the condition $\mu_{22}^{2}=\mu_{33}^{2}=-\mu_{23}^{2} \equiv \mu^{2}>0$, the mass spectrum in Eqs. (6)-(8) is as follows: only the third scalar in each sector becomes heavier since its mass gains a contribution of $\mu^{2}$

$m_{h_{1}}^{2}=m_{h}^{2}=\frac{2}{3} \lambda_{4} v_{\mathrm{SM}}^{2}, \quad m_{h_{2}}^{2}=\mu_{d}^{2}+\frac{1}{2} \bar{\lambda}^{\prime} v_{\mathrm{SM}}^{2}$,

$m_{h_{3}}^{2}=\mu_{d}^{2}+\frac{1}{2} \bar{\lambda}^{\prime} v_{\mathrm{SM}}^{2}+\mu^{2}, \quad m_{a_{1}}^{2}=0$,

$m_{a_{2}}^{2}=\mu_{d}^{2}+\frac{1}{6} \bar{\lambda}^{\prime} v_{\mathrm{SM}}^{2} \quad m_{a_{3}}^{2}=\mu_{d}^{2}+\frac{1}{6} \bar{\lambda}^{\prime} v_{\mathrm{SM}}^{2}+\mu^{2} \quad m_{c_{1}}^{2}=0$,

$m_{c_{2}}^{2}=\frac{1}{2} \mu_{d}^{2}+\frac{\lambda_{5}}{12} v_{\mathrm{SM}}^{2}, \quad m_{c_{3}}^{2}=\frac{1}{2} \mu_{d}^{2}+\frac{\lambda_{5}}{12} v_{\mathrm{SM}}^{2}+\mu^{2}$,

and the mass degeneracy in the inner sector has been broken but it is still possible an accidental degenerate with the SM-like Higgs scalar.

The mixing matrix remains the same as in Eq. (5).

In model $\mathrm{B}$, when the soft terms are included with the condition $\mu_{22}^{2}=\mu_{33}^{2}=\nu^{2}$, and $\mu_{23}^{2}=\mu^{2}$, we have

$\bar{m}_{h_{1}}^{2}=\lambda_{4} v_{\mathrm{SM}}^{2}, \quad \bar{m}_{h_{2}}^{2}=\mu_{d}^{2}+\frac{1}{2} \bar{\lambda}^{\prime} v_{\mathrm{SM}}^{2}+2 \mu^{2}-\nu^{2}$,

$\bar{m}_{h_{3}}^{2}=\mu_{d}^{2}+\frac{1}{2} \bar{\lambda}^{\prime} v_{\mathrm{SM}}^{2}+2 \mu^{2}+\nu^{2}, \quad \bar{m}_{a_{1}}^{2}=0$,

$m_{a 2}^{2}=\mu_{d}^{2}+\frac{1}{2} \bar{\lambda}^{\prime} v_{\mathrm{SM}}^{2}+2 \mu^{2}-\nu^{2}$,

$m_{a 3}^{2}=\mu_{d}^{2}+\frac{1}{2} \bar{\lambda}^{\prime} v_{\mathrm{SM}}^{2}+2 \mu^{2}+\nu^{2}, \quad \bar{m}_{c_{1}}^{2}=0$,

$m_{c_{2}}^{2}=\frac{1}{4}\left(2 \mu_{d}^{2}+\lambda_{5} v_{\mathrm{SM}}^{2}\right)+\mu^{2}-\frac{1}{2} \nu^{2}$,

$m_{c_{3}}^{2}=\frac{1}{4}\left(2 \mu_{d}^{2}+\lambda_{5} v_{\mathrm{SM}}^{2}\right)+\mu^{2}+\frac{1}{2} \nu^{2}$,

and the mixing matrix between the respective components of $\mathrm{H}_{2}$ and $\mathrm{H}_{3}$ is

$$
U=\left(\begin{array}{ccc}
1 & 0 & 0 \\
0 & -\frac{1}{\sqrt{2}} & \frac{1}{\sqrt{2}} \\
0 & \frac{1}{\sqrt{2}} & \frac{1}{\sqrt{2}}
\end{array}\right),
$$

and the mixing between $\mathrm{H}_{2}$ and $\mathrm{H}_{3}$ sector is maximal. In this case $S$ is still as in Eq. (9) but now

$$
\begin{aligned}
& D_{1}=\frac{1}{\sqrt{2}}\left(\begin{array}{c}
-h_{2}^{+}+h_{3}^{+} \\
\frac{1}{\sqrt{2}}\left(-h_{2}^{0}-i a_{2}^{0}+h_{3}^{0}+i a_{3}^{0}\right)
\end{array}\right), \\
& D_{2}=\frac{1}{\sqrt{2}}\left(\begin{array}{c}
h_{2}^{+}+h_{3}^{+} \\
\frac{1}{\sqrt{2}}\left(h_{2}^{0}+i a_{2}^{0}+h_{3}^{0}+i a_{3}^{0}\right)
\end{array}\right) .
\end{aligned}
$$


[1] ATLAS Collaboration, Phys. Lett. B 710, 49 (2012); CMS Collaboration, ibid.710, 30 (2012).

[2] G. C. Branco, P. M. Ferreira, L. Lavoura, M. N. Rebelo, M. Sher, and J. P. Silva, Phys. Rep. 516, 1 (2012).

[3] S. Pakvasa and H. Sugawara, Phys. Lett. 73B, 61 (1978); A. Mondragón, M. Mondragón, and E. Peinado, Phys. Rev. D 76, 076003 (2007); G. Bhattacharyya, P. Leser, and H. Päs, Phys. Rev. D 83, 011701 (2011); S. Kaneko, H. Sawanaka, T. Shingai, M. Tanimoto, and K. Yoshioka, Prog. Theor. Phys. 117, 161 (2007).

[4] A. C. B. Machado, J.C. Montero, and V. Pleitez, Phys. Lett. B 697, 318 (2011); R. de Adelhart Toorop, F. Bazzocchi, L. Merlo, and A. Paris, J. High Energy Phys. 03 (2011) 035.

[5] A.C. B. Machado and V. Pleitez, arXiv:1205.0995.

[6] C. C. Nishi, Phys. Rev. D 74, 036003 (2006); 76, 119901 (E) (2007); I. P. Ivanov, Phys. Lett. B 632, 360 (2006); Phys. Rev. D 75, 035001 (2007); 76, 039902(E) (2007); 77, 015017 (2008); P.M. Ferreira, H.E. Haber, M. Maniatis, O. Nachtmann, and J. P. Silva, Int. J. Mod. Phys. A 26, 769 (2011).

[7] I. P. Ivanov and E. Vdovin, Phys. Rev. D 86, 095030 (2012).

[8] G. Bhattacharyya, P. Leser, and H. Päs, Phys. Rev. D 83, 011701(R) (2011); 86, 036009 (2012); T. Teshima, Phys. Rev. D 85, 105013 (2012).

[9] N.G. Deshpande and E. Ma, Phys. Rev. D 18, 2574 (1978).

[10] M. Gustafsson, E. Lundström, L. Bergström, and J. Edsjo, Phys. Rev. Lett. 99, 041301 (2007); Phys. Rev. D 86, 075019 (2012); E. Lundstrom, M. Gustafsson, and J. Edsjo, Phys. Rev. D 79, 035013 (2009).
[11] For the representations and rules of the $S_{3}$ symmetry, see H. Ishimori, T. Kobayashi, H. Ohki, Y. Shimizu, H. Okada, and M. Tanimoto, Prog. Theor. Phys. Suppl. 183, 1 (2010).

[12] U. Ellwanger, J. High Energy Phys. 03 (2012) 044.

[13] J. F. Gunion, Y. Jiang, and S. Kraml, Phys. Lett. B 710, 454 (2012).

[14] J.F. Gunion, Y. Jiang, and S. Kraml, Phys. Rev. D 86, 071702(R) (2012).

[15] J. F. Gunion, Y. Jiang, and S. Krami, arXiv:1208.1817; A. Drozd, B. Grzadkowski, J. F. Gunion, and Y. Jiang, arXiv:1211.3580.

[16] P. M. Ferreira, R. Santos, H.E. Haber, and J.P. Silva, arXiv:1211.3131.

[17] ATLAS Collaboration, Eur. Phys. J. C 72, 2157 (2012).

[18] M. J. Matthew, C. Englert, and M. Spannowsky, J. High Energy Phys. 10 (2012) 112.

[19] G. Belanger, U. Ellwanger, J.F. Gunion, Y. Jiang, and S. Kraml, arXiv:1208.4952; ATLAS Collaboration, Report No. ATLAS-CONF-2012-091; CMS Collaboration, Report No. CMS-PAS-HIG-12-015.

[20] O. J. P. Eboli and D. Zeppenfeld, Phys. Lett. B 495, 147 (2000); R. M. Godbole, M. Guchait, K. Mazumdar, S. Moretti, and D. P. Roy, Phys. Lett. B 571, 184 (2003); D. Ghosh, R. Godbole, M. Guchait, K. Mohan, and D. Sengupta, arXiv:1211.7015.

[21] R. Barbieri, L. J. Hall, and V. S. Rychkov, Phys. Rev. D 74, 015007 (2006).

[22] Q.H. Cao, E. Ma, and G. Rajasekaran, Phys. Rev. D 76, 095011 (2007).

[23] F. Bazzocchi and M. Fabbrichesi, arXiv:1207.0951.

[24] CMS Collaboration, J. High Energy Phys. 09 (2012) 111. 